Cahiers $d u$ MONDE RUSSE

\section{Cahiers du monde russe}

Russie - Empire russe - Union soviétique et États indépendants

45/3-4 | 2004

Varia

\title{
I. A. Hristoforov, « Aristocratičeskaja » oppozicija Velikim reformam
}

\section{Wladimir Berelowitch}

\section{OpenEdition \\ Journals}

Édition électronique

URL : https://journals.openedition.org/monderusse/4183

DOI : 10.4000/monderusse.4183

ISSN : $1777-5388$

\section{Éditeur}

Éditions de l'EHESS

\section{Édition imprimée}

Date de publication : 1 juillet 2004

Pagination : 648-651

ISBN : 2-7132-2009-2

ISSN : $1252-6576$

Référence électronique

Wladimir Berelowitch, «I. A. Hristoforov, « Aristocratičeskaja » oppozicija Velikim reformam », Cahiers du monde russe [En ligne], 45/3-4 | 2004, mis en ligne le 11 février 2010, consulté le 03 septembre 2022. URL : http://journals.openedition.org/monderusse/4183 ; DOI : https://doi.org/10.4000/ monderusse. 4183

Ce document a été généré automatiquement le 3 septembre 2022

2011 


\title{
I. A. Hristoforov, «Aristocratičeskaja » oppozicija Velikim reformam
}

\author{
Wladimir Berelowitch
}

\section{RÉFÉRENCE}

\section{A. HRISTOFOROV, « Aristocratičeskaja » oppozicija Velikim reformam. Konec}

1850-seredina 1870 gg. (L'opposition « aristocratique » aux Grandes Réformes. Fin des années 1850-milieu des années 1870). Moscou, Russkoe slovo, 2002, 430 p.

$1 \quad \mathrm{Y}$ a-t-il eu un "parti » aristocratique en Russie qui se serait opposé aux Grandes Réformes d'Alexandre II, et si oui, quel fut-il? C'est à cette question, récurrente apparemment, que s'efforce de répondre Igor Hristoforov dans son premier ouvrage qui, à n'en pas douter, fera date par sa nouveauté dans l'historiographie de cette période.

2 Neuve, cette recherche l'est à de nombreux titres. Par sa base documentaire d'abord. I. Hristoforov ne s'est pas contenté de réexaminer les nombreux matériaux déjà publiés, notamment tous ceux qui s'attachent à l'abolition du servage, par exemple les volumineux Matériaux des Commissions de rédaction (Materialy Redakcionnyh komissij), les Journaux des comités gouvernementaux des affaires paysannes ainsi que les nombreux mémoires, correspondances et journaux intimes déjà publiés. Il s'est plongé dans une masse très impressionnante d'archives pratiquement inexploitées : journaux des comités provinciaux de la noblesse, mémoires, notes, correspondances adressés par différentes personnalités aux instances supérieures, et notamment au Comité en chef des affaires paysannes (Glavnyj komitet po krest'janskomu delu), papiers et correspondances conservés dans les fonds des ministres de l'Intérieur Lanskoj et Valuev, des frères Miljutin, du prince Čerkasskij, du comte Vladimir Petrovič Orlov-Davydov, des Bobrinskie, matériaux de la Commission pour la réforme des institutions des provinces et des districts, chargée de la réforme des zemstva, etc. Bien des documents ont été ainsi étudiés pour la première fois, 
par exemple le journal du comte Orlov-Davydov, les parties inédites du fameux journal de Dmitrij Miljutin, ou encore cette note, citée en annexe et en tout point remarquable, du prince A. I. Vasil'čikov, consacrée à la police secrète en Russie.

3 La nouveauté de l'ouvrage tient aussi à son sujet et à la façon dont l'auteur le traite. Comme on le sait, l'abolition du servage a donné lieu à une bibliographie abondante, tant en URSS, avec par exemple les ouvrages de Zajončkovskij, Družinin, Zaharova, que dans les pays occidentaux, dans les travaux de Terence Emmons, de George Yaney ou de Daniel Field. Dans son introduction, puis à de nombreuses reprises au cours de ses développements, l'auteur critique un certain nombre de stéréotypes qui s'étaient formés ici et là, et dont l'origine est souvent intéressante. Le principal d'entre eux était qu'un parti de nobles " conservateurs », partisans du servage (krepostniki), se fût opposé, non sans une certaine efficacité, à la réforme. Cette idée fut particulièrement répandue parmi les auteurs soviétiques, qui voyaient derrière l'action des réformateurs la main fort peu visible d'une révolution bourgeoise, tandis que les suppôts du servage représentaient selon eux des vestiges encore vivaces du monde féodal. L'auteur montre fort bien que ce cliché ne venait pas seulement des orientations idéologiques propres à l'historiographie de la période soviétique; il provenait, en amont, de l'étiquette que les adversaires directs des " aristocrates", les "bureaucrates éclairés» tels Nikolaj Miljutin (selon l'heureuse expression de Warner Bruce Lincoln dans son ouvrage du même titre), et les slavophiles comme Čerkasskij - évitons le terme de « libéraux » qui est trop flou et anachronique - leur avaient attribuée. C'est ainsi que l'expression «droits seigneuriaux » (votčinnye prava), qui avait été un cheval de bataille des "conservateurs", avait été dénoncée par les libéraux comme une preuve de leur " féodalisme ", alors qu'il s'agissait en fait pour eux de défendre le droit de propriété contre les intrusions de l'état.

Hristoforov s'intéresse donc à un groupe, mieux vaudrait dire à une mouvance ou à une constellation, de personnages proches de la cour. Certains d'entre eux étaient présents au sein du Conseil de l'Empire ou du Sénat et jouissaient de la sympathie de très hauts responsables comme Šuvalov et Valuev, ou encore le maréchal Barjatinskij. Outre les noms d'Orlov-Davydov, des Bobrinskie, on trouve ceux de N. A. Bezobrazov, de A. I. Platonov, de V. A. Dolgorukov, de P. P. Gagarin, etc., qui exercèrent fréquemment une influence à la tête ou au sein d'assemblées de la noblesse. La période étudiée court des débuts de la réforme agraire en 1857 jusqu'en 1875, soit après le limogeage (en 1874) du comte Petr Andreevič Šuvalov, chef des gendarmes et de la III ${ }^{e}$ section, et l'un des chefs de file de l'opposition " aristocratique».

5 Ce groupe, Hristoforov en analyse excellemment les logiques et les orientations qu'on pourrait résumer par trois traits : la répugnance à voir l'État intervenir dans les affaires rurales, autrement dit dans les rapports entre seigneurs et paysans; une certaine inspiration libérale (au sens vrai du mot, tel qu'on l'utilise en Occident) qui repose sur une défense de la propriété individuelle, noble bien sûr, mais aussi paysanne; et enfin le désir de poursuivre l'affranchissement de la noblesse en lui insufflant un esprit d'autonomie et en obtenant pour elle la haute main sur l'administration des districts ruraux, non pas en tant qu'agents de l'État mais sur le modèle de la gentry anglaise qui était leur référence explicite. Comme on le voit, on est loin d'un programme de maintien du servage. Cette mouvance ne mettait pas en doute la nécessité de la réforme, elle en contestait les grands choix : le fait qu'elle soit réalisée sous la contrainte - et donc avec intervention de l'État -, l'affranchissement de la paysannerie de la tutelle nobiliaire, et pis encore son assujettissement à la tutelle communautaire avec, au-dessus, encore une 
fois, celle de l'État. En même temps, les opposants « aristocratiques » savaient fort bien qu'on ne pouvait affranchir les paysans sans leur donner des terres à exploiter, sous peine de déclencher un véritable séisme social, et c'est cette nécessité (qui guida les réformateurs dans leurs choix) qui plaça l'opposition dans des contradictions à peu près insolubles. Comme l'écrit l'auteur, et sans doute à juste titre, les choix devant lesquels étaient placés les réformateurs étaient en réalité très étroits, si étroits qu'on peut à peine parler de choix.

6 Le propos de Hristoforov excelle par sa prudence, son sens des nuances et sa justesse de ton, ce qui ne l'empêche pas, comme on l'a vu, de s'attaquer à un certain nombre d'idées reçues. L'une des grandes originalités de ses analyses est qu'elles s'appuient non pas seulement sur les positions exprimées par les uns et les autres, mais aussi sur les jugements que les acteurs portaient sur leurs amis et ennemis dans leurs correspondances, journaux et mémoires. Cette méthode lui permet de cerner au plus près les réseaux de relations au sein et autour de la cour et aussi de tester les positions des uns et des autres par le regard d'autrui. L'une des questions les plus délicates qu'il a eu ainsi à résoudre était de mesurer le degré de cohésion et de force de l'opposition " aristocratique ». Sur ce plan, l'auteur ne souscrit pas à la thèse des "deux partis", « conservateur » et « libéral » qui auraient divisé la noblesse (c'était un peu la vision de T. Emmons dans son ouvrage), mais refuse aussi de croire que l'opposition " aristocratique » eût été un épouvantail, en grande partie fabriqué par les réformateurs pour servir leurs besoins tactiques (D. Field). Fortement inscrite dans la chronologie, son étude permet de mesurer les progrès de l'opposition, ses tentatives d'implantation dans les assemblées provinciales de la noblesse, ses succès relatifs après le tournant " conservateur» de 1866 et sa défaite quasi définitive après 1875 puisque, comme il le démontre avec une grande perspicacité, le programme des conservateurs des années 1880 (défense de la communauté rurale) était diamétralement opposé à celui de l'opposition « aristocratique » des années 1850-1870 qui détestait la communauté.

7 Cette mouvance était-elle effectivement «aristocratique »? Ce problème, bien que très clairement posé par l'auteur dans son introduction, reste un peu oublié dans ses développements, peut-être parce que, comme il l'écrit lui-même, il n'est guère facile de distinguer un tel groupe du reste de la noblesse. Bien entendu il existe bien, au sein de celle-ci, un ensemble de familles qui sont à la fois pourvues de grandes richesses foncières et qui occupent des postes clés dans l'armée et la haute administration. Mais l'ouvrage de I. Hristoforov tend à montrer que si l'opposition "aristocratique» se recrutait principalement dans ce groupe, elle ne le recoupait guère et que surtout, son "aristocratisme» relevait davantage d'un idéal politique (l'Angleterre telle que ces « aristocrates » la voyaient) que d'un intérêt structuré et ancré dans des pratiques sociales. La mouvance « conservatrice " (mais peut-on lui appliquer ce terme si ses idéaux relevaient d'un véritable bouleversement politique en Russie?) n'était finalement guère plus unie, guère plus cohérente que les « aristocrates » de 1730 ou des années 1760 : à cet égard, le très neuf et excellent ouvrage de I. Hristoforov prolonge de façon décisive les meilleures études de la noblesse russe, qui fut toujours en mal de son affranchissement, même lorsque l'actualité commandait celui de ses paysans. 